\title{
Retour sur les
}

origines de la vie

De l'atome aux molécules,

> Regarder plus de trois milliards d'années en arrière est difficile et la reconstruction d'arbres de l'évolution à partir de l'ADN actuel repose sur des hypothèses cachées qui ne permettent pas de retrouver ses vraies racines. Cherchant à s'affranchir de notre anthropocentrisme, le scénario proposé dans les deux textes qui seront successivement publiés écarte pour commencer l'idée d'une origine unique pour le remplacer par un scénario d'évolution qui ferait apparaître un processus réplicatif - formation d'une copie exacte - au sein d'un système chimique qui ne fait que se reproduire, formant des copies voisines de ce qu'il est. Les premières cellules formeraient une population de prédateurs assimilant peu à peu divers compartiments où se déroule la suite des étapes ancestrales. Échappant aux cellules prédatrices, deux types nouveaux, peu compartimentés, bactéries et archées seraient alors apparus pour envahir la Terre, former des organites au sein des prédateurs ancestraux en donnant la vie telle qu'on la connaît aujourd'hui. <

Apparaissant soudain sur Terre, chacun d'entre nous s'interroge sur ses origines. Au cours de cette quête, toute nouvelle analyse nous conduit à une origine unique. Nous avons tendance à être «adamistes $»^{1}$. L'astrophysicien britannique Fred Hoyle (1915-2001), explorant l'origine explosive de l'Univers, l'a nommée «Big Bang » par dérision, parce qu'il n'y croyait pas. Pourtant elle est désormais largement acceptée. La plupart des biologistes parleront aussi de LUCA, le last universal common ancestor anglais, imaginé par Christos Ouzounis et ses collègues (alors à l'EBI, The European Bioinformatics Institute, EMBL Cambridge

${ }^{1}$ La plupart des mythes d'origine imaginent un ancêtre unique à toute chose. II en est ainsi de l'Adam biblique. Par extension, nous pouvons dire que la pensée originelle est spontanément «adamiste ». En réalité, aucune espèce n'apparaît d'un seul coup. Elle résulte de l'émergence et de l'accumulation progressive de nouveaux caractères. Nous avons retenu ici l'un d'entre eux à l'origine de l'Homme, la fusion de deux chromosomes.

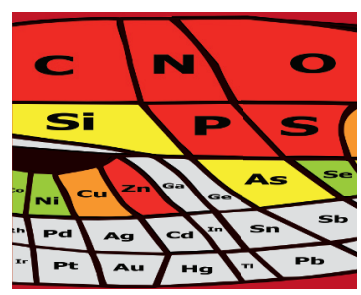

Institut de Cardiométabolisme et Nutrition, Hôpital de la PitiéSalpêtrière, 47, boulevard de l'Hôpital 75013 Paris, France. antoine.danchin@ normalesup.org

Outstation, Cambridge, Royaume-Uni), comme l'ancêtre commun à toutes les cellules vivantes [1,2]. De même, nous pensons que I'homme est apparu d'un coup au sein d'un groupe particulier de primates, ancêtres des singes actuels et des êtres humains. Est-ce bien raisonnable? En parallèle, nous sommes aussi victimes de l'idée que la «qualité » des êtres vivants peut être hiérarchisée, avec des êtres évolués et d'autres qui le sont moins. Cela rejoint un anthropocentrisme qui imagine qu'une grande taille va de pair avec le raffinement des comportements. Et pourtant, nous savons tous que ce raisonnement ne tient pas.

Si l'on regarde l'histoire des ordinateurs par exemple, ceux-ci n'ont cessé de diminuer en taille. L'ENIAC (electronic numerical integrator and computer $)^{2}$, qui ne faisait pas grand-chose, était un objet géant alors que nos téléphones cellulaires sont aujourd'hui aussi puissants que d'anciens très gros ordinateurs, comme les CRAY ${ }^{3}$ de naguère. De fait, l'ensemble des ordinateurs n'a cessé d'évoluer, chacun, qu'il soit supercalculateur ou téléphone, avec des propriétés toujours nouvelles et une taille toujours plus petite.

Pour comprendre les origines de la vie, renonçons donc à l'idée que les microbes sont des organismes simples et peu évolués. D'ailleurs, ils restent le futur de la vie sur Terre, bien après que plantes et animaux auront cessé de vivre en raison de chaleurs torrides ou de glaciations

${ }^{2}$ L'ENIAC est le premier ordinateur créé en 1946 par deux chercheurs américains de la Moore School, John W Mauchly et J Presper $\varepsilon c k e r t$. II pesait 30 tonnes, occupait $160 \mathrm{~m}^{2}$ et contenait 170468 tubes électroniques, 70000 résistances et 6000 commutateurs qu'il fallait tourner manuellement avant de brancher les centaines de câbles nécessaires à l'exécution d'une simple opération.

${ }^{3}$ CRAY est le nom d'une entreprise américaine, fondée en 1972 par Seymour Cray, qui conçoit et fabrique des superordinateurs. Le CRAY-1 est lancé en 1976. II s'agit d'un superordinateur à architecture vectorielle. II est construit autour d'un processeur 64 bits cadencé à $83 \mathrm{MHz}$, doté de 8 Mo de mémoire vive et refroidi au fréon. 


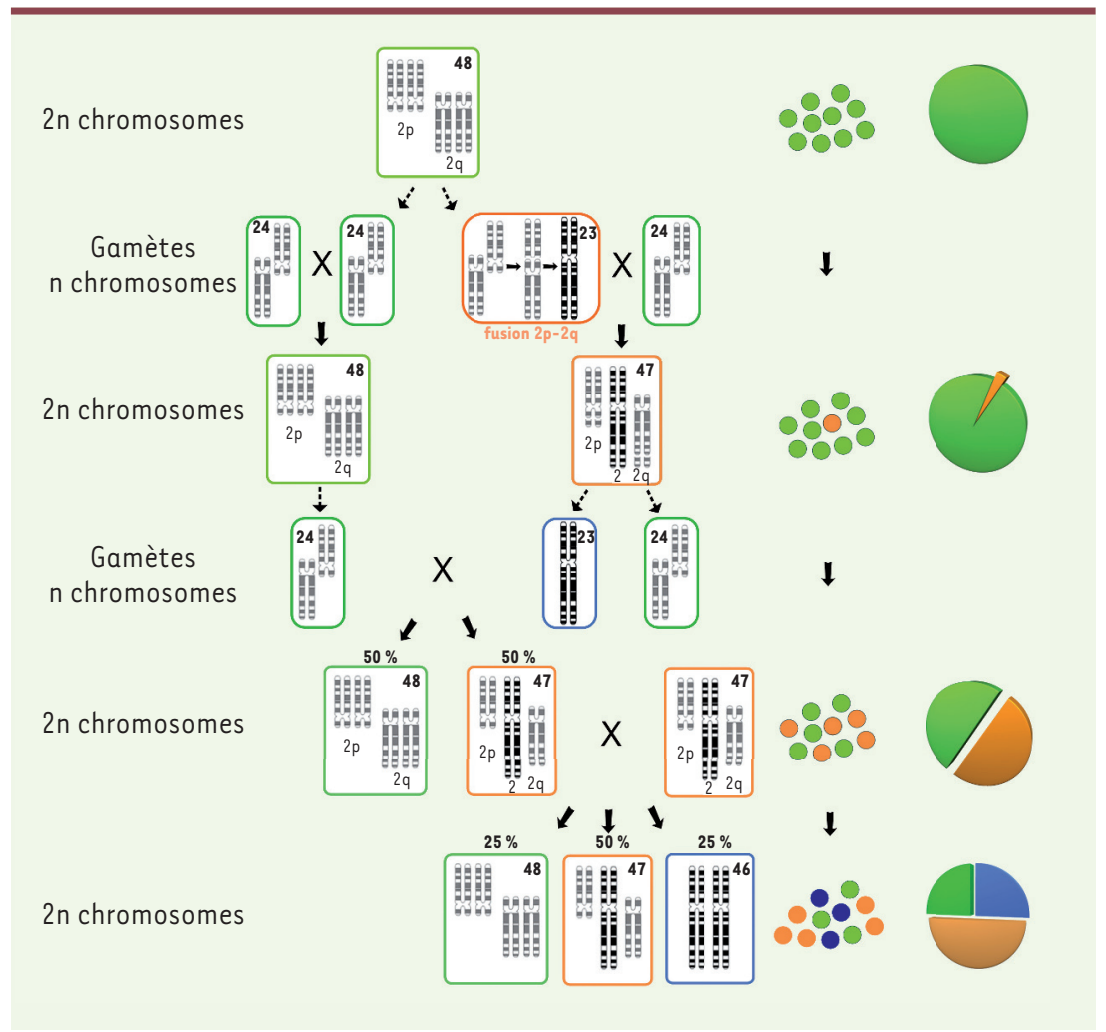

Figure 1. L'homme est issu d'un remarquable accident génétique. Un accident génétique spectaculaire, la fusion de deux chromosomes, va enclencher le processus qui donnera naissance à l'homme moderne au sein d'une population de primates qui évoluera aussi vers les grands singes. Pour en arriver à ce que nous sommes il a fallu de nombreuses générations, de nombreux mélanges, qui ont fait passer le nombre de nos chromosomes de 48 à 46 , via une étape instable à 47 chromosomes.

ancêtres a produit, à la suite d'une fusion accidentelle, un gamète fertile contenant 23 chromosomes - notre chromosome 2 fusion - au lieu de 24, comme chez ses parents. Bien sûr, le croisement avec un individu normal conduisait à une descendance porteuse d'une anomalie génétique, avec 47 chromosomes. Ce nombre impair est instable: au cours de la méiose, la double division qui conduit aux gamètes, va en donner un mélange à 23 et 24 chromosomes.

terrifiantes, comme cela s'est déjà produit plusieurs fois au cours de I'histoire de la Terre. Tentons ici d'abandonner nos préjugés.

\section{Détour par les origines de l'homme moderne}

La quête d'une origine unique est si profondément enracinée dans nos esprits, que j'ai choisi de dépeindre l'origine de l'homme moderne comme modèle d'origines floues. C'est que le doute s'est installé récemment... Les choses sont un peu plus compliquées que le récit traditionnel faisant soudain apparaître l'espèce humaine. En effet, les Européens modernes sont un mélange d'Homo sapiens, venu d'Afrique, mais aussi d'Homo neanderthalensis, à l'origine incertaine, africaine elle aussi. Les personnes originaires d'Asie et surtout d'0céanie ont en outre la trace d'un ou plusieurs Homo denisova ${ }^{4}$ dans leurs gènes. Les Tibétains ont même extrait des membres de cette espèce d'hominiens, une version d'un gène, EPSAl (endothelial PAS domain-containing protein 1), qui leur a permis de prospérer au-dessus de $4500 \mathrm{~m}$. Mais voici une observation remarquable: ces ancêtres du genre Homo avaient probablement comme nous 46 chromosomes, à la différence des grands singes (chimpanzés, bonobos, gorilles, orang-outans) qui en comptent 48 . Cela pose la question simple : comment, d'un ancêtre commun, avons-nous pu passer de 48 à 46 chromosomes?

Nos gènes sont si semblables à ceux de nos cousins que nous savons que notre chromosome 2 résulte de la fusion tête-bêche de deux chromosomes individuels chez les grands singes. II faut donc imaginer un accident génétique spectaculaire à l'origine de l'homme moderne. Un de nos

${ }^{4}$ Des gènes de plusieurs espèces d'Homo denisova ont été identifiés chez les individus d'0céanie.
Si sa fertilité n'est pas trop affectée, cet individu, ne rencontrant que des congénères «normaux», produira une descendance dont la moitié aura le «bon» nombre de 48 chromosomes, et l'autre moitié, 47 chromosomes. Les hominidés sont des animaux sociaux. Cela conduira donc à une colonie conséquente d'individus des deux sexes ayant 47 chromosomes. Naturellement, des individus de ce type se sont alors accouplés. Leur progéniture est alors singulière : elle comprend des sujets semblables à ceux du groupe initial (à 47 et 48 chromosomes), mais aussi des sujets bien différents, porteurs d'un plus petit nombre de chromosomes, pair et donc stable (46, et en supposant I'hérédité standard mendélienne avec les proportions: $1 / 4$ $48,1 / 247$ et $1 / 446$ ). Mais les individus à 46 chromosomes pouvaient alors s'accoupler avec n'importe lequel des membres du groupe! Aussi, après plusieurs générations, une colonie stable de membres à 46 chromosomes a pu commencer à se développer, s'adaptant à ce nouveau génome, vraisemblablement au cours de nombreuses générations (Figure 1). Nulle part Adam ou Ève: cette origine ne peut que s'étaler durant des siècles. C'est bref aux échelles géologiques, bien sûr, et peut faire croire $a$ posteriori et de façon erronée à un événement soudain.

\section{Reproduction et réplication}

L'origine des premières cellules est tout aussi floue. Pour le comprendre, nous devons commencer par nous 


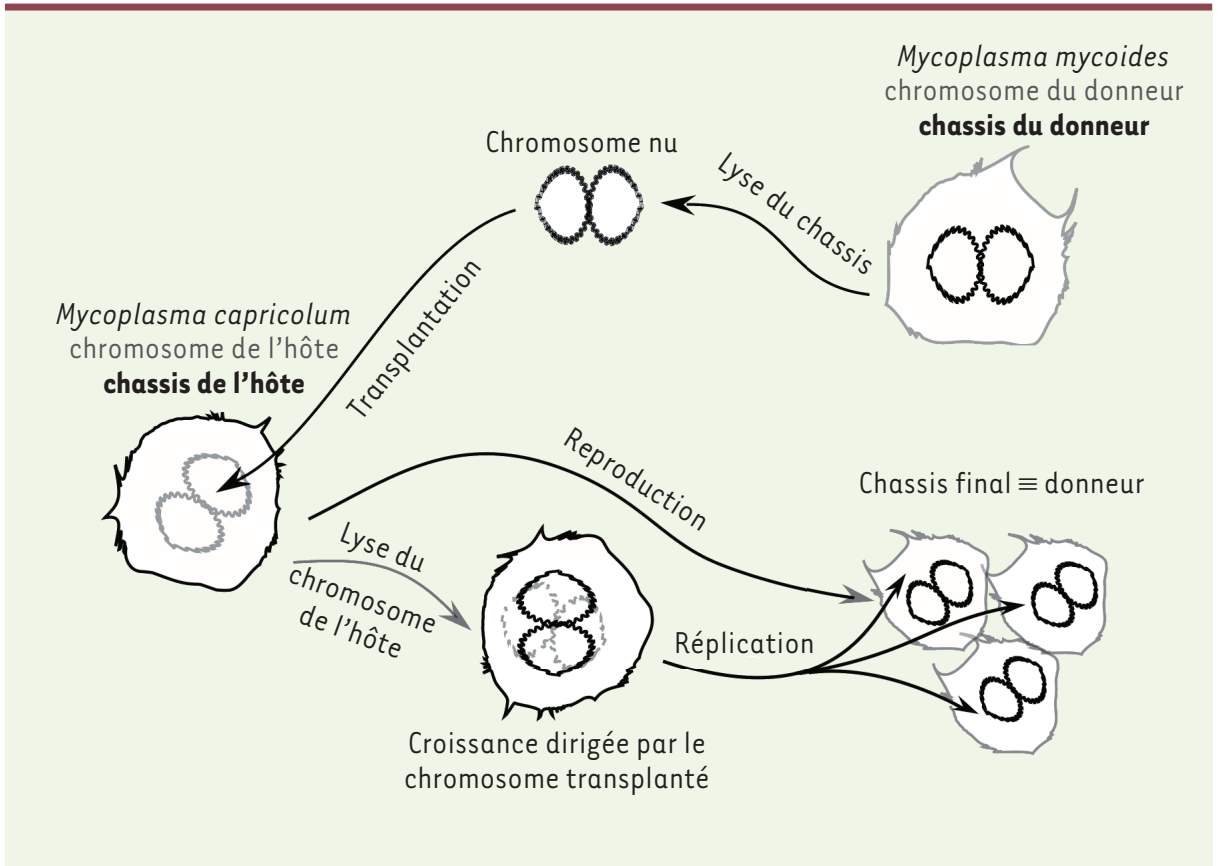

Figure 2. Conséquences de la transplantation d'un génome entier: reproduction et réplication. Le chromosome extrait d'une espèce de Mycoplasme est transplanté dans une cellule d'une autre espèce. Ce chromosome a été modifié de façon à coder la synthèse d'une enzyme qui détruira le chromosome originel de son hôte. II va alors s'y substituer tout en se répliquant et peu à peu remplacer les composants de I'hôte par d'autres, identiques à ceux de l'espèce donneuse. À la fin de l'expérience, on observe une colonie bactérienne où les chromosomes sont tous identiques au chromosome transplanté, alors que la machinerie qui en exprime le programme sera différente de celle de l'hôte. faire une idée de ce qu'est la vie. L'exercice est difficile. C'est que ses lois sont abstraites et obscures, profondément cachées dans le concret de la matière et toutes ses idiosyncrasies. Aussi, malgré ses principes universaux, la vie telle qu'on peut l'observer est submergée par les anecdotes. Pour comprendre ses lois, nous aurons donc à faire un aller-et-retour constant entre le concret et l'abstrait. Cela commence ainsi : deux lois, développées en trois processus, définissent le domaine de la physico-chimie des organismes vivants.

Un premier processus impose que leurs composants soient compartimentés: l'atome de vie est la cellule, définissant un milieu intérieur distinct de ce qui l'entoure. Ensuite, intervient le temps. Les composants chimiques cellulaires donnent lieu à de multiples interconversions, constituant son métabolisme. Pour agir, ce processus construit et utilise des fonctions dont le rôle est de manipuler les objets biologiques. Concrètement, cette manipulation est réalisée pour l'essentiel par des molécules géantes (ou macromolécules) de la classe des protéines. Enfin, la cellule est en mesure de transmettre «quelque chose », d'une génération à la suivante. Ce processus de mémorisation lui permet d'assurer une certaine pérennité à sa structure et à son comportement. Une autre classe de macromolécules, celle des acides nucléiques, le permet également. Ces opérations sont fondées sur l'application de deux lois capitales. La première identifie, puis sélectionne et utilise la complémentarité entre structures matérielles (un peu à la manière d'une serrure et de sa clé, ou du positif et du négatif d'une photographie argentique). La seconde en est une conséquence majeure, très abstraite et profonde. Elle exploite une règle de codage (à la manière du chiffrement des messages secrets) qui permet de passer, de façon imposée, d'une structure matérielle (celle des acides nucléiques, dont I'ADN est le type) à une autre structure matérielle (celle des protéines).
La question qui nous occupe est donc de concevoir des scénarios physico-chimiques qui, en implémentant les deux lois centrales, conduisent aux trois processus clés. Certains auteurs se sont émerveillés de l'ensemble des conditions qu'il fallait supposer réunies. C'est que, sans divinité organisant le monde, la mise en place simultanée de cet ensemble n'est pas vraisemblable. Mais bien sûr il n'y a aucune raison que ce que nous voyons aujourd'hui réuni en un seul ensemble soit né d'un seul coup. C'est ainsi que le célèbre physicien américanoanglais Freeman Dyson, dans un petit livre publié en 1985 , Origins of Life, établit sans ambiguïté que la vie doit découler d'au-moins deux origines. II imagine d'abord un système chimique, ancêtre du métabolisme cellulaire d'aujourd'hui, qui se reproduit, construisant des copies approximatives, mais capables de produire un sous-ensemble restant fertile. Une fois cette première étape bien établie, Dyson imagine qu'une de ces copies fait émerger en son sein des sous-structures qui se répliquent (c'est-à-dire qu'elles produisent des copies exactes d'elles-mêmes). Cela permet de pérenniser la mémoire d'une partie du système, mais en ralentit l'évolution (notons au passage que la réplication, processus physique, ne peut échapper aux erreurs, bien plus rares toutefois que celles de l'étape de reproduction qui la précède). Cette distinction entre reproduction et réplication, qui est capitale, reste malheureusement ignorée de la plupart des réflexions sur les origines de la vie. En effet, bien des auteurs ont une vue presque magique, et en tout cas animiste, de cette origine. Ils mettent en avant une sorte de pouvoir créateur 
spécifique de telle ou telle catégorie de composants chimiques ou de principes physiques. Pourtant, la nécessité d'une double origine permet de comprendre comment un sous-ensemble du monde chimique a pu se trouver regroupé au sein de structures cellulaires bien définies, et a pu évoluer vers la vie. Soumises à une sélection très forte-seules les proto-cellules ayant une descendance ne disparaissaient pas -, celles-ci se reproduisent de plus en plus efficacement. Elles retiennent alors les molécules-clés qui vont développer et enrichir la loi de complémentarité. Ces dernières deviennent à la fois aptes à se répliquer et à faire émerger les conditions d'apparition de la loi de codage qui, aujourd'hui, fait passer des acides nucléiques aux protéines.

Nous remarquons ici la dualité profonde qui rend compte de la vie. Une cellule vivante est une machine qui se reproduit tout en répliquant son programme de construction. La destination finale de ce programme génétique, le génome formé d'acides nucléiques, est de produire une copie aussi exacte que possible de lui-même, au sein d'une copie approximative de la machine qui l'exécute. Comment les processus de copie du programme et de copie de la cellule sont-ils reliés ? En termes matériels, les choses diffèrent. La réplication utilise aujourd'hui l'ADN, acide nucléique particulièrement stable. La double hélice, qui constitue cette molécule étonnante, a capturé de façon exemplaire une première loi de complémentarité assurant la conservation pérenne du programme. Au contraire, la cellule ne sait produire d'elle-même que des copies approximatives, mais non identiques. Deux échelles de temps sont associées à cette dichotomie : la réplication est fiable durant de nombreuses générations, tandis que la reproduction fait des copies qui varient d'une génération à la suivante.

Il s'agit bien d'une différence cruciale, illustrée de façon éloquente dans l'expérience spectaculaire de la transplantation du génome d'une cellule d'une espèce au sein d'une autre espèce [3] (Figure 2). À la fin de l'expérience, le génome extrait de colonies bactériennes en croissance est identique à celui qui a été transplanté initialement dans l'hôte. En revanche, la machinerie qui lit le programme, et même la forme de la cellule, diffère dans l'hôte initial et dans les cellules des colonies finales. La machine parentale s'est multipliée et ses composants ont été remplacés par d'autres, voisins, mais non identiques. Le processus de multiplication a commencé par utiliser l'ancienne machinerie, mais n'a pu que se servir du programme transplanté comme notice de construction de ces nouveaux objets. Or ce programme est différent du programme parental, détruit au cours de la transplantation. Il a donc produit des composants de la machine qui ne sont pas les mêmes que ceux de l'hôte initial.

Nous sommes familiers de ce fait: issus d'un œuf unique, les tissus d'un animal sont bien différenciés. Leur génome est pourtant le plus souvent parfaitement identique au génome initial. C'est d'ailleurs ce qui permet de «reprogrammer» les cellules pour leur permettre de former un tissu autre que le tissu de départ. En bref, alors que le programme reste le même, la machine qui l'exécute est assez variable. Elle conserve cependant ses principales propriétés fonctionnelles (abstraites) : lire et exprimer le programme, diriger la construction d'une descendance, surveiller l'état de l'environnement, extraire les ressources propres et éliminer ou réparer les composants inutiles ou usés. La relation entre la machine et le programme est au cœur de cette interaction essentielle. C'est l'origine de cette relation qui constitue la question clé qui nous occupe.

\section{Reproduction d'un ensemble dynamique de réactions chimiques dans un compartiment}

Revenons maintenant à la matière. Imaginons comment se sont construites les unités de base que l'on retrouve aujourd'hui dans toutes les cellules de toutes les espèces et, en particulier, les deux classes centrales de macromolécules déjà évoquées, protéines et acides nucléiques. Toutes sortes de scénarios en rendent compte, depuis la chaleur de sources sous-marines [4], celle des volcans [5], les décharges électriques des orages [6], le rayonnement ultraviolet [7], mais aussi l'eau maintenue en surpression dans la glace [8], la surface des pierres [9], l'alternance de périodes sèches et pluvieuses [10] et une infinité de variantes. Ces scénarios sont rarement compatibles entre eux. Ils s'ignorent les uns les autres. Quelques réactions chimiques, ici ou là, semblent plausibles. II n'existe cependant pas de modèle cohérent qui puisse à la fois expliquer la genèse de tous les composants essentiels de la vie actuelle et l'élimination sélective de ceux qui se sont nécessairement formés en parallèle. D’un fouillis général, il a fallu en effet extraire un petit sous-ensemble fonctionnel. Cela fait apparaître pour la première fois la sélection naturelle, à savoir éliminer ce qui est nuisible à la production d'une descendance tout en gardant jalousement tout ce qui fonctionne, même maladroitement.

\section{Fermeture}

Parmi tous ces scénarios, ceux qui conduisent à la genèse de compartiments cellulaires bien différenciés explorent des pistes qui ont un avenir intéressant. L'eau, fluide aux propriétés uniques, s'impose toujours comme hôte de la chimie prébiotique. Ce fluide a la particularité de favoriser la compartimentation de molécules en fonction de leur caractère chimique: hydrophile, aisément soluble, ou hydrophobe, tendant à s'écarter du volume du fluide et donc à créer des compartiments, justement ce que nous avons retenu comme premier processus essentiel. De nos jours, ces cellules sont entourées de feuillets faits d'une double couche de lipides, molécules allongées hydrophiles à une extrémité et hydrophobes à l'autre. Cela leur permet de prendre une structure tête-bêche, conduisant à un feuillet plan [11]. C'est là qu'apparaît une première singularité physique spontanée et centrale pour 


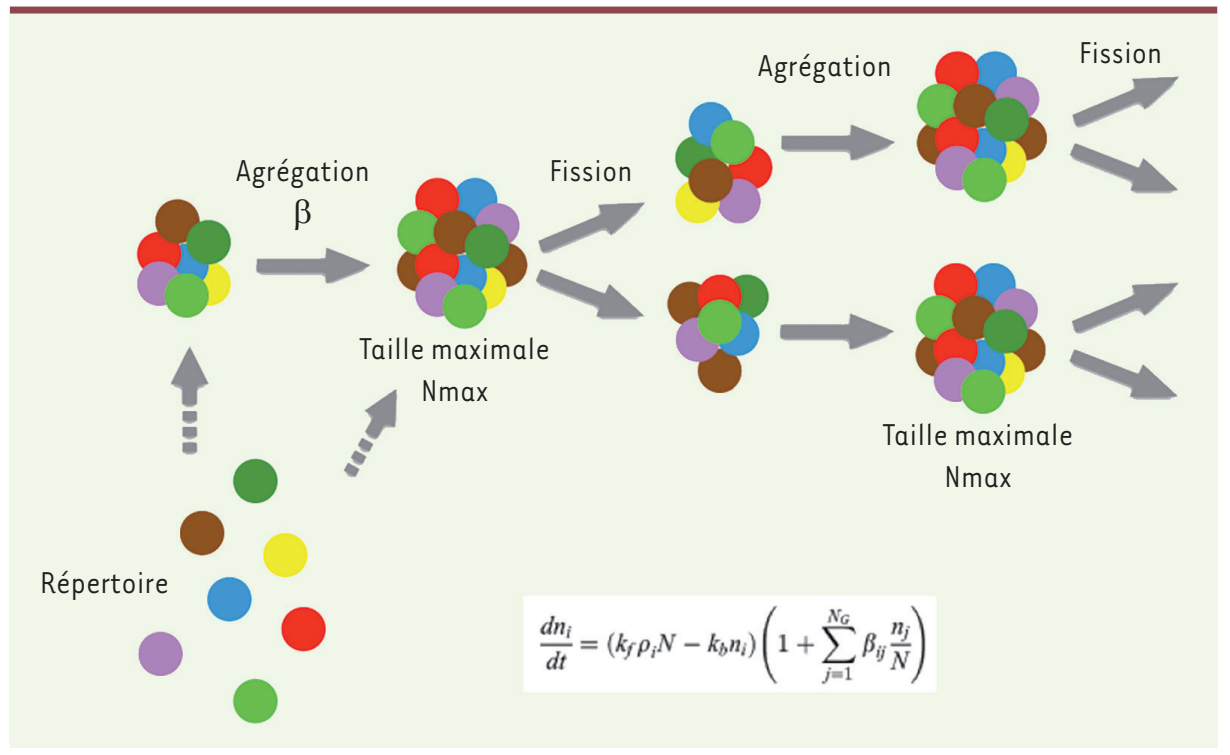

Figure 3. Schéma d'évolution par reproduction compositionnelle (GARD). Différents types de molécules (représentés par des cercles colorés différents) s'associent et forment des agrégats de composition moyenne constante. Le processus d'agrégation est régi par une matrice de vitesses liée à la complémentarité des molécules qui interagissent. Une fois qu'un assemblage atteint une certaine taille (Nmax), il se divise. Sa descendance reprend un cycle de croissance (génération après génération). Au cours de ce processus, la composition des assemblages devient de plus en plus stable dans le temps. C'est au sein de tels assemblages que peuvent apparaître des structures proprement réplicatives (transmises à l'identique d'une génération à la suivante) (inspiré de [20]).

la genèse des cellules. Soumis à une agitation inévitable, ces feuillets, qui sont très flexibles, se déforment, et il arrive un moment où leurs bords se rencontrent, formant une vésicule. Cette fusion accidentelle rompt la symétrie du milieu en faisant apparaître, naturellement, une structure aux propriétés émergentes, séparant un intérieur de l'extérieur, et donc capable d'évolution distincte, ... une cellule primitive. C'est la première création d'une information nouvelle, cruciale pour la suite.

\section{Réseaux de réactions chimiques qui se reproduisent}

Dans ces vésicules, des molécules peuvent interagir, former et détruire de nouvelles molécules. Les vésicules peuvent aussi grossir, se scinder, et englober d'autres vésicules, formant ainsi un emboîtement de compartiments. Certains chercheurs [12], pour s'affranchir des idiosyncrasies de la matière, ont construit des modèles abstraits fondés sur la compartimentation, mais sans faire référence explicite à des molécules particulières, retenant simplement leurs propriétés de complémentarité qui leur permettent d'interagir. Cela conduit à la formation de réseaux moléculaires au sein de vésicules, séparées mais capables de fusionner, de se scinder tour à tour, et même de s'engloutir. Les auteurs de ces modèles proposent qu'en leur sein des assemblages protocellulaires sont produits, aidés par l'action catalytique d'éléments de l'ensemble. II y a donc au moins trois types de composants: ceux qui permettent la compartimentation, ceux qui forment le réseau d'interactions, et ceux qui favorisent ou empêchent cette formation.
Divers composés amphiphiles et hydrophobes constituent naturellement les premiers systèmes capables de stockage, d’héritage et de canalisation de l'information correspondante [13]. Ce qui est intéressant dans ces modèles est qu'avec des propriétés de reconnaissance minimales, donc plausibles, on peut concevoir un système évoluant en produisant de nouveaux ensembles semblables aux premiers, qui s'améliorent peu à peu.

Ces modèles du type GARD (graded autocatalysis replication domain) (Figure 3) reposent sur la présence en leur sein, de composés permettant la catalyse de transformations métaboliques et de nouveaux assemblages stables, tout en assurant leur propre pérennité [14]. Malgré leur malheureux nom (leurs domaines se reproduisent, ne se répliquent pas!), ces modèles illustrent très bien la première étape du scénario de Dyson, qui met l'accent sur la reproduction. Leurs réseaux d'interactions conduisent, tout naturellement, à penser que certaines de ces molécules peuvent réagir les unes avec les autres et en former de nouvelles [15]. Cela nous impose de revenir à la matière et à la chimie qui lui correspond.

\section{Des atomes de la vie aux composants du métabolisme cellulaire}

Passer de la simple reproduction à la fidélité de la réplication suppose le stockage stable de la mémoire du passé. II faut aussi des effecteurs des fonctions cellulaires. Cela est réalisé chez les organismes vivants par la synthèse des acides nucléiques et des protéines, molécules géantes formées de motifs chimiques de base qui sont extraits d'un petit ensemble. Les construire est chimiquement très contraignant.

\section{Atomes}

Dans l'eau liquide, à la température de la Terre, cela suppose en effet la formation de liaisons covalentes (partage d'électrons) entre les atomes. Les atomes qui forment les molécules du vivant ne peuvent donc être quelconques: faire des édifices stables et complexes 
requiert une bonne réactivité chimique, une abondance assez grande et une grande stabilité moléculaire. Ce sont les orbitales externes des atomes qui fusionnent pour donner des molécules. L'écrantage du noyau par les couches électroniques internes rend instables la plupart des édifices macromoléculaires formés d'atomes lourds. Ainsi, il ne subsiste guère que l'hydrogène, le bore, le carbone, l'azote, l'oxygène et le fluor comme atomes possibles pour la synthèse des macromolécules complexes, stables et diversifiées (Figure 4).

C'est ensuite l'abondance de ces éléments qui va déterminer leur présence dans les macromolécules: le bore est rare dans l'univers, on ne le trouvera donc pas comme constituant majeur de la vie; le fluor, combiné au carbone, donne des molécules bien trop stables pour permettre un métabolisme efficace aux températures où l'eau est liquide. II n'est pas non plus présent de façon significative. De fait, les atomes élémentaires de la vie sont donc l'hydrogène, le carbone, l'azote et l'oxygène. D'autres s'y ajoutent néanmoins. Les réactions chimiques supposent une panoplie d'échanges d'électrons, et le soufre, analogue plus lourd de l'oxygène et abondant sur Terre, a été retenu à cet effet. En parallèle, la gestion de l'énergie nécessaire à la vie utilise une propriété unique du phosphore. Formant des phosphates, cet atome peut conserver de l'énergie dans des liaisons phosphoester qui sont métastables dans l'eau : bien qu'elles soient naturellement décomposées par l'eau, il faut beaucoup d'énergie pour les rendre accessibles à cette dégradation. II reste enfin toute une série d'atomes qui perdent les électrons de leur couche externe et sont utilisés sous forme d'ions, pour la catalyse et la gestion du potentiel électro-chimique de la cellule.

\section{Unités de base}

Cinq familles de molécules, formées de quelques dizaines d'atomes («petites » molécules, métabolites), ont été retenues au sein des premières cellules: les acides aminés, les lipides, les sucres, les nucléotides, et les cofacteurs de la catalyse des réactions biochimiques [16]. En fonction de leur préférence pour tel ou tel scénario, la plupart des auteurs n'en gardent vraiment que deux, les acides aminés et les nucléotides, oubliant la nécessité d'expliquer aussi les autres. Certaines sont combinées sous la forme de macromolécules, faites de millions, voire de milliards d'atomes. Il est néanmoins logiquement inévitable que le monde des petites molécules ait précédé celui des macromolécules. Nous avons considéré ici deux classes, les protéines, enchaînements d'une vingtaine d'acides aminés comme motifs de base via une liaison chimique originale, la liaison peptidique, et les acides nucléiques, enchaînements de quatre motifs, les nucléotides, via une liaison, dite phosphodiester, impliquant le phosphate. Ces enchaînements s'écrivent de façon symbolique comme un texte (leur « séquence ») utilisant vingt lettres pour les protéines, et quatre pour les acides nucléiques. La synthèse des petites molécules, acides aminés des protéines et nucléotides des acides nucléiques, constitue la partie primordiale du métabolisme, celle qui a précédé la synthèse des macromolécules. Pour cela, elle utilise plusieurs cofacteurs essentiels pour la catalyse de réactions réalisée par une immense famille de protéines, les enzymes. Ignorant le raisonnement de Dyson, la controverse fait rage entre ceux qui tiennent à la présence initiale de polypeptides et ceux qui préfèrent les acides nucléiques, spécialement les ARN. II faut en effet des protéines pour construire I'ARN... mais l'ARN est nécessaire au codage et à la synthèse des protéines. Nous avons là typiquement un dilemme du type de celui de savoir qui, de l'œuf ou la poule, préexiste [17].

Pourtant, ce qu'un scénario d'origine doit expliquer, c'est à la fois la genèse des composants de base et la mise à l'écart de ceux qui leur ressemblent au point d'empoisonner les réactions dans lesquelles ils sont impliqués. Cela suppose comprendre la logique du métabolisme [18]. Les acides aminés des protéines, pour beaucoup d'entre eux, sont faciles à synthétiser et stables. Bien d'autres existent dans la cellule, et ceux des protéines sont loin d'être aléatoires. Ils obéissent à des contraintes quantitatives, chimiques et fonctionnelles. Plusieurs sont omniprésents dans l'univers (les plus petits). Ils peuvent être convertis, les uns en les autres, à un faible coût énergétique. Ils sont répartis selon trois propriétés physico-chimiques importantes liées à l'eau : hydrophilie, amphiphilie ou hydrophobie. Une fois retenus, la sélection naturelle a concrétisé les fonctions nécessaires pour écarter tous ceux qui leur ressemblent, soit chimiquement, soit par leur forme. Une exception très inattendue se présente alors à nous. Liée aux accidents de l'Histoire, elle est exemplaire du caractère anecdotique des contraintes matérielles de la vie: la proline, l'un des vingt constituants des protéines, n'est pas un acide aminé... C'est un iminoacide, aux propriétés chimiques bien différentes. Or sa présence, certainement très tôt, a eu des conséquences majeures pour la synthèse des protéines. Incorporer cet élément dans un polypeptide requiert une famille de fonctions spécifiques, créant alors, au cœur même de la cellule, une famille d'anecdotes qui vont nous cacher les règles abstraites de son fonctionnement. De même, la mécanique de la synthèse des protéines, qui se fonde sur des adaptateurs ARN, les ARN de transfert (ARNt), introduit une contrainte chimique qui écarte du processus plusieurs acides aminés pourtant naturellement présents dans les cellules, comme l'ornithine, l'homosérine ou I'homocystéine.

La question est encore plus aiguë pour les nucléotides, maillons de la chaîne des acides nucléiques. Ces molécules, et surtout les ribonucléotides des ARN, sont formées à partir du ribose, un sucre très réactif et instable, impossible à accumuler, et dont il faut par conséquent expliquer la genèse continue. L'origine des sucres, composants ubiquistes de la cellule, 


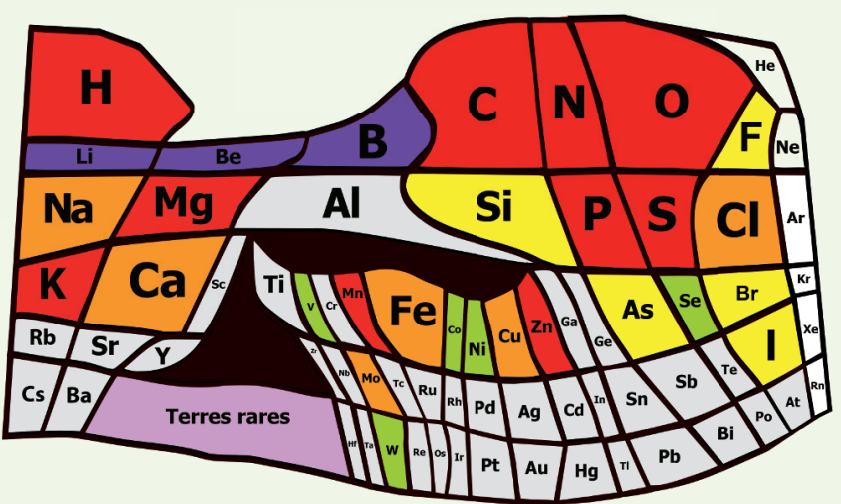

Figure 4. Les atomes de la vie. Une vue du tableau périodique des éléments où la surface associée à chaque atome est proportionnelle à son abondance sur la Terre (adapté de [21]). La vie se construit à partir d'atomes capables de former des molécules géantes à la température où l'eau est liquide. II s'y ajoute des atomes permettant les transferts d'électrons, la gestion du potentiel électrique et la catalyse, ainsi que un atome dont le rôle est original, le phosphore, au cœur de la gestion de l'énergie par la cellule. Ces atomes sont indiqués en rouge. En violet, on trouve des atomes rares dans l'Univers. En orange, les atomes présents très souvent chez les organismes vivants. On remarquera que le fer n'est pas omniprésent. Un certain nombre d'organismes en sont dépourvus (en particulier ceux qui ont été utilisés pour l'expérience de transplantation). Le chrome n'est pas retenu dans la liste. II s'agit d'un atome qui réagit spontanément avec les protéines (il est ainsi utilisé pour le tannage du cuir) et se retrouve donc systématiquement comme contaminant des cellules. En vert clair sont représentés des atomes encore très répandus et en jaune des atomes rarement présents. En blanc, on trouve les gaz rares, inertes chimiquement. Ces catégories sont susceptibles de changer (sauf la catégorie rouge) au fur et à mesure qu'on découvre de nouveaux organismes.

est d'ailleurs une question clé qui peut être résolue au moyen de scénarios qui rendent compte de la fixation du gaz carbonique dans les conditions de la Terre primitive [19]. Pour finir, l'origine des cofacteurs qui sont les véritables agents de la catalyse des réactions enzymatiques reste énigmatique, malgré sa nécessité. En dehors des complexes associant le fer et le soufre, naturellement omniprésents sur Terre, il n'existe pratiquement aucun scénario en expliquant l'origine [16].

\section{Stockage et utilisation de l'énergie : rôle central du phosphate} Le phosphate s'accumule sous la forme de polyphosphates, résistants à l'irradiation ou à la dessiccation. Cette propriété, qui permet aux composés phosphatés de stocker de l'énergie, explique pourquoi le phosphore est un atome essentiel de la vie. De multiples synthèses métaboliques utilisent des nucléotides comportant trois phosphates à la queue-leu-leu. II faut noter ce qui pourrait sembler une bizarrerie peu économe en énergie, mais ces composants libèrent souvent sous l'action de l'eau - une hydrolyse - un groupe de deux phosphates enchaînés (le pyrophosphate). Plus de cinq cents réactions produisent, aujourd'hui, du pyrophosphate. L'une d'entre elles est le moteur qui polymérise les nucléotides en acides nucléiques. Une autre est à l'origine de la synthèse des protéines. Or, comme presque toutes les réactions de la vie, ces réactions sont réversibles et proches de l'équilibre - au contraire de croyances communes. Aussi, la chaîne du polynucléotide en formation devrait bientôt cesser de s'allonger au fur et à mesure que le pyrophosphate s'accumule. Cela révèle une réaction critique pour la vie - c'est elle qui est son moteur principal. Coupant le pyrophosphate en deux phosphates à l'aide de l'eau en grand excès dans la cellule, les pyrophosphatases assurent la marche en avant de la vie.

\section{Capture d'information précoce : « la dissymétrie c'est la vie 》 (Louis Pasteur)}

Un attribut de la vie est qu'elle manipule de l'information. Aussi faut-il retracer les origines de sa capture progressive. Les quatre liaisons de l'atome de carbone sont orientées aux sommets d'un tétraèdre. Deux figures en miroir, où les sommets diffèrent, dites chirales, peuvent avoir exactement la même composition chimique. II s'agit de stéréoisomères. Retenir l'un ou l'autre apporte une information. Pour comprendre la spécificité des enzymes, il faut imaginer une clé, son miroir, et une seule serrure. Les acides aminés (sauf le plus simple d'entre eux, la glycine) existent sous deux formes principales: I'une, L- (pour lévogyre), est celle que l'on trouve dans les protéines. L'origine de cette première chiralité est un thème rebattu. S'il est pourtant sans grand intérêt, c'est que les ruptures de symétrie sont chose courante - on doit circuler sur une route ou à gauche, ou à droite - et l'origine de celle des acides aminés n'a aucune raison d'être autre que contingente. Cependant, une fois ce choix fait, il va cette fois imposer une longue suite de structures dissymétriques qui, elles, ne sont plus accidentelles. L'une d'entre elles a trait aux sucres, fondés sur une symétrie de la famille D- (pour dextrogyre). Ces molécules dérivent en effet d'une molécule à trois atomes de carbone, le glycérate. II en existe deux stéréoisomères, L- et D-. Or le L-glycérate ressemble très fortement à un acide aminé essentiel, la L-sérine. Comme les sucres sont abondants dans la cellule, il va de soi que du L-glycérate en excès, ou ses dérivés, vont parasiter les processus impliquant la L-sérine, en particulier la synthèse des protéines et celle des membranes cellulaires. Le $\mathrm{D}$-glycérate est donc devenu le stéréoisomère préféré comme en conséquence la plupart des sucres. Le ribose et le désoxyribose sont ainsi du type D-. 


\section{Conclusion}

Au contraire d'Athéna, sortie entière et armée de la cuisse de Jupiter, aucune cellule vivante n'est apparue soudainement. Un ensemble de réactions chimiques, peu à peu coordonnées, a dû précéder la genèse des macromolécules, protéines et acides nucléiques qu'on reconnaît aujourd'hui au cœur de la vie. II a fallu d'abord que ces ensembles primitifs, réunis au sein de vésicules se scindant et fusionnant tour à tour, se reproduisent en une descendance semblable mais non identique à ses parents. Ces premières structures cellulaires ont commencé à faire émerger une qualité particulière du monde physique, de l'information - catégorie physique illustrant ce qu'est la mémoire - dans un ensemble encore peu stable, faute d'être capable de mémoriser ce qui dirige sa composition. C'est la découverte en son sein du processus de réplication (formation d'une copie exacte d'une mémoire liée à l'organisation de la cellule) qui sera l'objet de la deuxième partie de ce scénario. $\diamond$

Revisiting the origins of life: from atoms to molecules, reproduction, then replication

\section{LIENS D'INTÉR̂ิET}

L'auteur déclare n'avoir aucun lien d'intérêt concernant les données pubiées dans cet article.

\section{RéFÉRENCES}

1. Ouzounis C, Kyrpides N. The emergence of major cellular processes in evolution. FEBS Lett 1996 ; $390: 119-23$

2. Kyrpides $\mathrm{N}$, Overbeek $\mathrm{R}$, Ouzounis $\mathrm{C}$. Universal protein families and the functional content of the last universal common ancestor.J Mol Evol $1999 ; 49: 413-23$.

3. Lartigue C, Glass JI, Alperovich N, et al. Genome transplantation in bacteria: changing one species to another. Science $2007 ; 317: 632-8$.

4. Westall F, Hickman-Lewis K, Hinman N, et al. A hydrothermal-sedimentary context for the origin of life. Astrobiology $2018 ; 18: 259-93$.

5. Fryer P. Serpentinite mud volcanism: observations, processes, and implications. Ann Rev Mar Sci $2012 ; 4: 345-73$.
6. Bada JL. New insights into prebiotic chemistry from Stanley Miller's spark discharge experiments. Chem Soc Rev 2013 ; 42 : 2186-96.

7. Michaelian K. Microscopic dissipative structuring and proliferation at the origin of life. Heliyon $2017 ; 3$ : e00424.

8. Feller G. Cryosphere and psychrophiles: Insights into a cold origin of life? Life (Basel) $2017 ; 7$.

9. Wachtershauser $\mathrm{G}$. The origin of life and its methodological challenge.J Theor Biol 1997 ; 187 : 483-94.

10. Deamer $D$. Conjecture and hypothesis: The importance of reality checks. Beilstein J Org Chem 2017 ; $13: 620$-4.

11. Deamer D. Membranes and the origin of life: a century of conjecture. J Mol Evol $2016 ; 83$ : 159-68.

12. Blokhuis $A$, Lacoste $D$, Nghe $P$, Peliti L. Selection dynamics in transient compartmentalization. Phys Rev Lett 2018 ; 120 : 158101. doi: 10.1103/ PhysRevLett.120.158101.

13. Segre D, Lancet D. Composing life. EMBO Rep $2000 ; 1: 217-22$.

14. Segre $D$, Lancet $D$, Kedem 0 , Pilpel Y. Graded autocatalysis replication domain (GARD): kinetic analysis of self-replication in mutually catalytic sets. Orig Life Evol Biosph 1998 ; 28 : 501-14.

15. Shapiro R. Small molecule interactions were central to the origin of life. $\varrho$ Rev Biol $2006 ; 81: 105-25$.

16. Sharov AA. Coenzyme world model of the origin of life. Biosystems 2016; $144: 8-17$.

17. Danchin A. L'CFuf et la Poule. Histoires du Code Génétique. Paris : Fayard, $1983: 282 \mathrm{p}$.

18. Danchin A, Sekowska A. The logic of metabolism and its fuzzy consequences. Environ Microbiol $2014 ; 16: 19-28$.

19. Smith $\varepsilon$, Morowitz HJ. Universality in intermediary metabolism. Proc Natl Acad Sci USA 2004 ; 101 : 13168-73.

20. Segre D, Ben-Eli D, Lancet D. Compositional genomes: prebiotic information transfer in mutually catalytic noncovalent assemblies. Proc Natl Acad Sci USA $2001 ; 97: 219-30$.

21. Sheehan WF. Periodic table of elements with emphasis. Chemistry 1976; 49 : 17-8.
TIRÉS À PART

A. Danchin

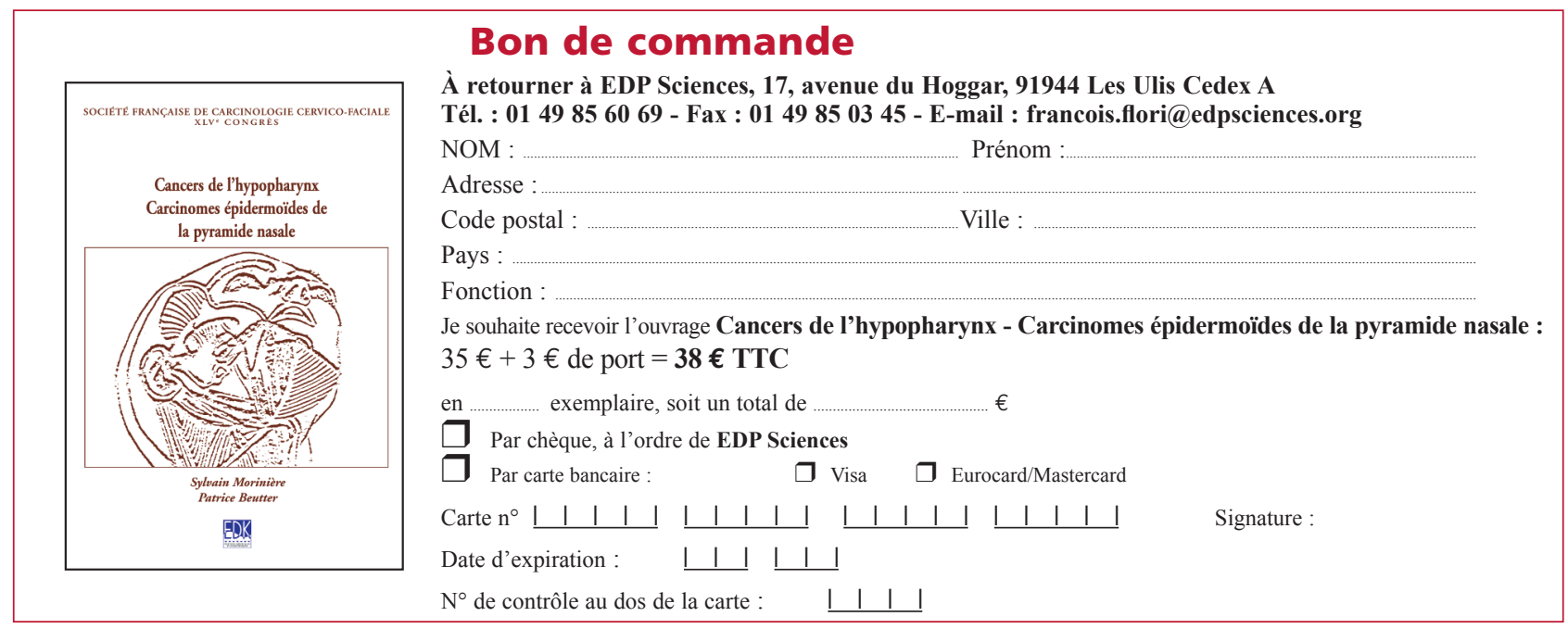

